\title{
Intraday Trading Patterns in the SSE 50 ETF Option
}

\author{
Xu Tongtong ${ }^{1, a, *}$, Wang Susheng ${ }^{1, b}$, Peng $\mathrm{Ke}^{1, \mathrm{c}}$, You Dandan ${ }^{1, \mathrm{~d}}$, and Hu Mingzhu ${ }^{1, \mathrm{e}}$ \\ ${ }^{1}$ School of Economics and Management, Harbin Institute of Technology, Shenzhen 518055, China. \\ axtt87090@163.com, b786210107@qq.com, '406946393@qq.com, dyoudandan620@yahoo.com, ${ }^{\mathrm{e}} 7$ \\ 69994192@qq.com
}

\begin{abstract}
The purpose of this article is to explore the Shanghai Stock Exchange (SSE) 50 ETF Option's intraday trading patterns which include return and volatility. We get the SSE 50 ETF option's intraday trading patterns by charts, descriptive statistics and Wilcoxon rank sum test. We find that the call option's return has no obvious intraday pattern and the put option's return roughly follows an LM-shaped pattern; The call option's volatility roughly follows an L-shaped pattern and the put option's volatility roughly follows an LM-shaped pattern.
\end{abstract}

Keywords: the SSE 50 ETF Option, intraday trading patterns, return, volatility.

\section{Introduction}

To explain the intraday patterns in financial market, scholars have developed several micro-structure theories. With the development of electronic trading systems, the availability of high frequency data is greatly improved. Presently, it is hot to research the market microstructure by using high-frequency data.

\section{Literature Review}

The 50 ETF Option was launched on February 9, 2015 and is traded on Shanghai Stock Exchange. The 50 ETF Option whether has intraday trading patterns, what the intraday trading patterns are and what factors cause the intraday trading patterns are interesting problems that need to be solved urgently.

Various studies have researched the intraday trading patterns in different market. Peterson (1990) examines the return intraday patterns in call options and put options traded on the Chicago Board Options Exchange (CBOE). He find that call options have relatively high returns late in the trading day and put options have low returns late in the trading day. Sheikh and Ronn (1994) reveal an intraday U-shaped pattern in the variances of adjusted option returns in CBOE. Tian and Guo (2007) find an L-shaped intraday return volatility pattern in the Shanghai Composite Stock Index. Gordon and Karen (2010) examine The Tracker Fund of Hong Kong's intraday premiums patterns and they find the pattern of the measures (range, SD and number of transactions of the Tracker Fund of Hong Kong premiums) follows either a double-U shape or an asymmetric $\mathrm{W}$-shape across the time interval of a day. Li et.al. (2012) find a U-shaped and an L-shaped intraday pattern for trading volume and return volatility in benchmark ETFs, leveraged ETFs, and leveraged inverse ETFs. Mishra and Daigler (2014) find the intraday trading patterns for two closely related index options-SPX and SPY are quite different, and they find trading measures, including returns, volume, bid-ask spreads, and volatility of SPY options show the U-shaped pattern, whereas SPX not.

Most previous researches reveal that the returns, volatility, and other microstructure variables have U-shaped intraday pattern. The SSE 50 ETF Option as China's first stock option was launched on 9 February and there is no research studying the intraday pattern of the SSE 50 ETF Option so far. This article will reveal the SSE 50 ETF Option's intraday trading patterns including the returns, and 
the volatility to fill the blanks as well as to provide a reference for investment decision-making and market regulations.

\section{Sample and Methods}

In this study, the sample is during 9 February, 2015 to 22 May, 2015 and the data is got from Shanghai Stock Exchange. The trading time in a day is from 9:30 to 11:30 and from 13:00 to 15:00. This article divides the trading time into sixteen successive 15-minute intervals. Using 15-minute intervals rather than 1-minute intervals or others is concerning that the new option's trading is not that active and this time interval allows us to obtain estimates of variables even for less actively trades options without much loss of data synchronicity. Besides, we roll over to the next nearest contract when it emerges as the most active contract to avoid thin markets and expiration effects.

Compound interest is used to calculate returns and the formula is as follows:

$$
r_{t}=\ln \left(P_{t} / P_{t-1}\right)
$$

Where $P_{t}$ is the close price in time period t; $P_{t-1}$ is the close price in time period t-1.

In order to contain more information, we use Garman-Klass (GK) statistic to measure volatility as Booth and Raymond do. The calculation method of GK is as formula (2):

$$
G K_{t}=1 / 2\left(P_{t}^{\text {High }}-P_{t}^{\text {Low }}\right)-(2 \ln 2-1)\left(P_{t}^{\text {Close }}-P_{t}^{\text {Open }}\right)^{2}
$$

Where $P_{t}^{\text {High }}$ is the natural logarithms of the highest price in time period t; $P_{t}^{\text {Low }}$ is the natural logarithms of the lowest price in time period t; $P_{t}^{\text {Close }}$ is the natural logarithms of the close price in time period $\mathrm{t} ; P_{t}{ }^{\text {Open }}$ is the natural logarithms of the open price in time period $\mathrm{t}$. The volume and the open interest can be get from the original data.

\section{Results and Discussion}

Fig.1 demonstrates the intraday pattern in returns of the SSE 50 ETF options for 15-minutes intervals in a trading day.

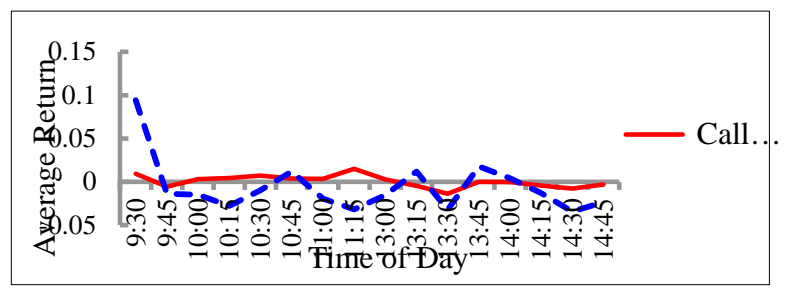

Fig.1 The average rate of return for each 15-minutes intervals of the trading day From the graphs, we can get that the average rate of return of the put options is more volatile than the call options. The return of put options is high at the open time and then it declines at 9:45-10:00. At the following time, it fluctuates below zero at most time. The return of call options fluctuates around zero in a small amplitude. Subsequent test is pending to check whether the phenomenon is general or it is affected by certain extreme data. Table 1 reports the descriptive statistics and the rank tests of returns of the SSE 50 ETF call and put options for each fifteen-minute interval in a trading day. 
Table 1 Return of the options

\begin{tabular}{|c|c|c|c|c|c|c|c|c|c|c|c|c|}
\hline \multirow{2}{*}{ Time } & \multicolumn{6}{|c|}{ The SSE 50 ETF Call Option } & \multicolumn{6}{|c|}{ The SSE 50 ETF Put Option } \\
\hline & Mean & Median & JB & $\mathrm{P}(\mathrm{JB})$ & Rank-sum & $\mathrm{P}(\mathrm{R}-\mathrm{S})$ & Mean & Median & JB & $\mathrm{P}(\mathrm{JB})$ & Rank-sum & $\mathrm{P}(\mathrm{R}-\mathrm{S})$ \\
\hline $9: 30$ & 0.010 & 0.026 & 12.8 & 0.00 & 1.12 & 0.26 & 0.094 & -0.025 & 8272.8 & 0.00 & -0.16 & 0.88 \\
\hline $9: 45$ & -0.006 & -0.022 & 286.1 & 0.00 & -1.42 & 0.16 & -0.014 & 0.004 & 166.4 & 0.00 & 1.58 & 0.11 \\
\hline 10:00 & 0.003 & -0.002 & 3.1 & 0.22 & 0.00 & 1.00 & -0.015 & -0.011 & 401.1 & 0.00 & 0.00 & 1.00 \\
\hline $10: 15$ & 0.004 & 0.009 & 55.0 & 0.00 & 0.78 & 0.44 & -0.028 & -0.002 & 456.7 & 0.00 & 0.71 & 0.48 \\
\hline $10: 30$ & 0.007 & 0.007 & 20.7 & 0.00 & 0.73 & 0.47 & -0.010 & -0.013 & 0.6 & 0.74 & 0.34 & 0.73 \\
\hline $10: 45$ & 0.004 & -0.004 & 1.2 & 0.54 & - & - & 0.012 & 0.000 & 1908.9 & 0.00 & 1.16 & 0.25 \\
\hline $11: 00$ & 0.004 & 0.007 & 0.6 & 0.75 & - & - & -0.019 & -0.015 & 543.6 & 0.00 & 0.26 & 0.80 \\
\hline $11: 15$ & 0.015 & 0.004 & 8.3 & 0.02 & 0.79 & 0.43 & -0.032 & -0.019 & 330.3 & 0.00 & -0.26 & 0.80 \\
\hline $13: 00$ & 0.003 & 0.003 & 129.3 & 0.00 & -0.09 & 0.93 & -0.015 & -0.001 & 142.4 & 0.00 & 0.47 & 0.64 \\
\hline $13: 15$ & -0.005 & 0.001 & 2.3 & 0.32 & - & - & 0.012 & 0.005 & 46.0 & 0.00 & 2.18 & 0.03 \\
\hline $13: 30$ & -0.014 & -0.015 & 26.4 & 0.00 & -1.61 & 0.11 & -0.032 & 0.001 & 4905.9 & 0.00 & 1.09 & 0.28 \\
\hline $13: 45$ & 0.000 & -0.004 & 53.0 & 0.00 & -0.38 & 0.71 & 0.018 & 0.000 & 3999.6 & 0.00 & 1.66 & 0.10 \\
\hline $14: 00$ & 0.000 & -0.003 & 78.1 & 0.00 & -0.39 & 0.70 & 0.004 & 0.000 & 8.4 & 0.02 & 1.51 & 0.13 \\
\hline $14: 15$ & -0.004 & 0.006 & 11.4 & 0.00 & 0.06 & 0.95 & -0.013 & -0.014 & 390.6 & 0.00 & 0.47 & 0.64 \\
\hline $14: 30$ & -0.008 & -0.005 & 334.7 & 0.00 & -0.50 & 0.62 & -0.034 & -0.001 & 3301.4 & 0.00 & 0.97 & 0.33 \\
\hline $14: 45$ & -0.003 & 0.000 & 30.4 & 0.00 & -0.39 & 0.70 & -0.023 & -0.007 & 867.2 & 0.00 & 0.54 & 0.59 \\
\hline
\end{tabular}

Since the probability of JB in time 10:00-11:15 and 13:15-13:30 for call option is bigger than 0.1, returns in these time intervals follow a normal distribution. Wilcoxon rank-sum test is not proper to test the difference between time 10:00-10:15 and other time interval. $T$ test is then used to test the difference between 10:00-10:15 and the other three time intervals and the result is as table 2 .

Table 2 Test for some call time intervals

\begin{tabular}{|c|c|c|c|}
\hline Time & & $\mathrm{T}$ Test & \\
\hline \multirow{2}{*}{$10: 45$} & Ha: mean(diff) $<0$ & Ha: mean(diff) $!=0$ & Ha: mean(diff) $>0$ \\
\hline & $\operatorname{Pr}(\mathrm{T}<\mathrm{t})=0.4727$ & $\operatorname{Pr}(|\mathrm{T}|>|\mathrm{t}|)=0.9454$ & $\operatorname{Pr}(\mathrm{T}>\mathrm{t})=0.5273$ \\
\hline \multirow{2}{*}{ 11:00 } & Ha: mean (diff) $<0$ & Ha: mean(diff) $!=0$ & Ha: mean(diff) $>0$ \\
\hline & $\operatorname{Pr}(\mathrm{T}<\mathrm{t})=0.4859$ & $\operatorname{Pr}(|\mathrm{T}|>|\mathrm{t}|)=0.9717$ & $\operatorname{Pr}(\mathrm{T}>\mathrm{t})=0.5141$ \\
\hline \multirow{2}{*}{ 13:15 } & Ha: mean (diff) $<0$ & Ha: mean(diff) $!=0$ & Ha: mean $($ diff $)>0$ \\
\hline & $\operatorname{Pr}(\mathrm{T}<\mathrm{t})=0.7213$ & $\operatorname{Pr}(|\mathrm{T}|>|\mathrm{t}|)=0.5575$ & $\operatorname{Pr}(\mathrm{T}>\mathrm{t})=0.2787$ \\
\hline
\end{tabular}

From the Jarque-Bera statistics, we can get that the call option's intraday returns follow a non-normal distribution in most time intervals. From Wilcoxon rank-sum test and the T test, we can get that all $p>0.1$. This shows that there is no significant difference between 10:00-10:15 and other time interval. Combined with Figure 1 and the statistical data, we can get that the call option's return has a slight fluctuation around zero. The return of the call option's intraday pattern is not obvious. Table 1 also shows that put returns in most time intervals except 10:30-10:45 follow a non-normal distribution. Then non-parametric test is suitable to test the difference between 10:00-10:15 and other time intervals. From the Wilcoxon rank-sum test, we can get that in time 9:45-10:00, 13:15-13:30 and 13:45-14:00, the return have significant difference with in time 10:00-10:15. Combined with the statistical data and Figure 1, we can see that in time 9:45-10:00, 13:15-13:30 and 13:45-14:00, the return is all higher than in time 10:00-10:15. The put option's return roughly follows an LM-shaped pattern. At the opening time in the morning, much overnight information is absorbed by the market and the market trading is active. The return is relatively high at the opening time in the morning. At the opening time in the afternoon, the investors take a wait-and-see attitude, so the trade is not that active which induces the return is unrealistically high.

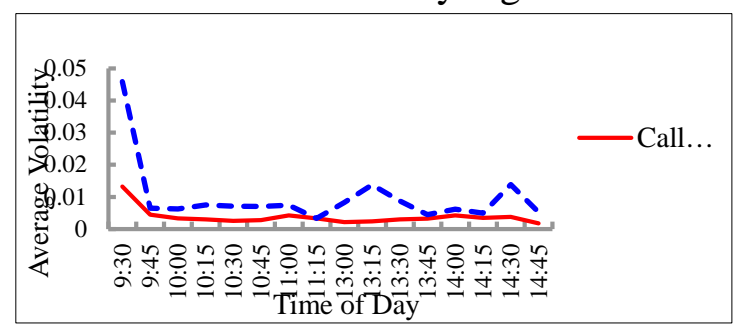

Fig.2 The average volatility for each 15-minutes intervals of the trading day

Fig.2 demonstrates the intraday pattern in GK volatility of the SSE 50 ETF options for fifteen-minute intervals in a trading day. From the graphs, we can get that the call and put options both have a high volatility at the opening time in the morning. The call option's volatility is relatively stable compared with the put option. Subsequent test is pending to check whether the phenomenon is general or it is affected by certain extreme data. 
Table 3 Volatility of the options

\begin{tabular}{|c|c|c|c|c|c|c|c|c|c|c|c|c|}
\hline \multirow{2}{*}{ Time } & \multicolumn{6}{|c|}{ The SSE 50 ETF Call Option } & \multicolumn{6}{|c|}{ The SSE 50 ETF Put Option } \\
\hline & Mean & Median & JB & $\mathrm{P}(\mathrm{JB})$ & Rank-sum & $\mathrm{P}(\mathrm{R}-\mathrm{S})$ & Mean & Median & JB & $\mathrm{P}(\mathrm{JB})$ & Rank-sum & $\mathrm{P}(\mathrm{R}-\mathrm{S})$ \\
\hline $9: 30$ & 0.013 & 0.005 & 861.7 & 0.000 & 4.51 & 0.000 & 0.046 & 0.005 & 11411.5 & 0.000 & 3.18 & 0.002 \\
\hline $9: 45$ & 0.005 & 0.002 & 9597.2 & 0.000 & 0.40 & 0.069 & 0.007 & 0.002 & 1692.5 & 0.000 & 0.41 & 0.679 \\
\hline $10: 00$ & 0.003 & 0.001 & 3179.4 & 0.000 & 0.00 & 1.000 & 0.006 & 0.002 & 975.6 & 0.000 & 0.00 & 1.000 \\
\hline $10: 15$ & 0.003 & 0.001 & 1631.5 & 0.000 & -0.87 & 0.384 & 0.008 & 0.001 & 1163.0 & 0.000 & -1.15 & 0.250 \\
\hline $10: 30$ & 0.003 & 0.001 & 125.3 & 0.000 & -1.19 & 0.235 & 0.007 & 0.001 & 7260.9 & 0.000 & -1.87 & 0.061 \\
\hline $10: 45$ & 0.003 & 0.001 & 136.7 & 0.000 & -0.13 & 0.898 & 0.007 & 0.002 & 1326.9 & 0.000 & -0.22 & 0.829 \\
\hline $11: 00$ & 0.004 & 0.002 & 1330.2 & 0.000 & 0.59 & 0.557 & 0.007 & 0.002 & 1838.5 & 0.000 & -0.14 & 0.891 \\
\hline $11: 15$ & 0.003 & 0.002 & 298.1 & 0.000 & 0.96 & 0.336 & 0.003 & 0.002 & 254.4 & 0.000 & -0.08 & 0.934 \\
\hline $13: 00$ & 0.002 & 0.001 & 830.7 & 0.000 & -1.55 & 0.122 & 0.008 & 0.001 & 7295.4 & 0.000 & -2.18 & 0.030 \\
\hline $13: 15$ & 0.002 & 0.001 & 1173.9 & 0.000 & -1.08 & 0.279 & 0.014 & 0.001 & 10575.5 & 0.000 & -1.26 & 0.207 \\
\hline $13: 30$ & 0.003 & 0.001 & 386.9 & 0.000 & -0.99 & 0.321 & 0.009 & 0.001 & 2547.5 & 0.000 & -0.86 & 0.392 \\
\hline $13: 45$ & 0.003 & 0.001 & 4589.6 & 0.000 & -0.53 & 0.598 & 0.004 & 0.001 & 4210.2 & 0.000 & -1.17 & 0.242 \\
\hline $14: 00$ & 0.004 & 0.002 & 4165.4 & 0.000 & 0.57 & 0.570 & 0.006 & 0.002 & 4875.1 & 0.000 & -0.24 & 0.807 \\
\hline $14: 15$ & 0.003 & 0.001 & 285.1 & 0.000 & -0.75 & 0.452 & 0.005 & 0.001 & 718.5 & 0.000 & -1.26 & 0.210 \\
\hline $14: 30$ & 0.004 & 0.002 & 449.3 & 0.000 & 0.93 & 0.354 & 0.014 & 0.001 & 6149.8 & 0.000 & -1.03 & 0.301 \\
\hline $14: 45$ & 0.002 & 0.001 & 170.9 & 0.000 & -1.98 & 0.048 & 0.005 & 0.001 & 1342.2 & 0.000 & -1.84 & 0.067 \\
\hline
\end{tabular}

Table 3 reports the statistics as well as the Wilcoxon rank-sum test of the volatility for call and put options. From the rank-sum test in table 4, we can get the volatility of call option in time 9:30-10:00 is quite different from time 10:00-10:15. Combined with the statistics, we find the volatility in 9:30-10:00 is large than 10:00-10:15. By the same method, we find the volatility in time 14:45-15:00 is slightly smaller than in time 10:00-10:15. The volatility of the call option roughly follows an L-shaped pattern. From the Wilcoxon rank-sum test in table 5, we can get that the volatility of the put option in time 9:30-9:45, 10:30-10:45, 13:00-13:15 and 14:45-15:00 is quite different from 10:00-10:15. Combined with the mean and median statistics, we find the volatility in 9:30-9:45, $10: 30-10: 45,13: 00-13: 15$ is larger than 10:00-10:15. The volatility in 14:45-15:00 is smaller than in time 10:00-10:15. The volatility of the put option roughly follows an LM-shaped pattern. At the opening time in the morning, much overnight information is absorbed by the market and the market trading is active. The volatility of call and put options are both large in this time. At the closing time, investors adjust their positions to avoid overnight risk that reduces the market volatility.

\section{Conclusions}

This paper examines the SSE 50 ETF Option's intraday trading patterns in return and volatility. Rather than U-shaped trading pattern as other markets', the SSE 50 ETF Option presents different intraday patterns. The return of the call option's intraday pattern is not obvious and the return of the put option roughly follows an LM-shaped pattern. The volatility of the call option roughly follows an L-shaped pattern and the volatility of the put option roughly follows an LM-shaped pattern. The volatility in the morning opening time is significantly higher than in other periods, which indicates that investors likely take the trading point and facilitate trading cluster. Investors can use the above intraday patterns to guide their investment decisions and regulators can use the above intraday patterns to monitor the market more effectively.

\section{Acknowledgment}

This work is supported by Shenzhen science and technology innovation plan, Shenzhen science and technology research and development funds and basic research project (Project No.: JCYJ20140417173156101).

\section{References}

[1] Peterson D R. a transaction data study of day-of-the-week and intraday patterns in option returns [J]. Journal of Financial Research, 1990, 13(2):117-131.

[2] Sheikh A M, Ronn E I. A Characterization of the Daily and Intraday Behavior of Returns on Options [J]. The Journal of Finance, 1994, 49(2):557-579.

[3] Tian G G, Guo M. Interday and intraday volatility: Additional evidence from the Shanghai Stock Exchange [J]. Review of Quantitative Finance and Accounting, 2007, 28(3):287-306. 
[4] Gordon Y. N. Tang, Karen H. Y. Wong. A note on the intraday and intraweek patterns in premiums of exchange-traded funds: evidence from Hong Kong [J]. Applied Economics Letters, 2010, 17(8):753-760.

[5] Li M, Klein D P, Zhao X. Empirical Analysis of ETF Intraday Trading [J]. Financial Services Review, 2012, 21.

[6] Mishra S, Daigler R T. Intraday Trading and Bid-Ask Spread Characteristics for SPX and SPY Options [J]. Journal of Derivatives, 2014, 21(21):70-84. 